PROCEEDINGS OF THE

AMERICAN MATHEMATICAL SOCIETY

Volume 140, Number 4, April 2012, Pages 1221-1232

S 0002-9939(2011)11270-X

Article electronically published on October 18, 2011

\title{
NOTES ON REGULARITY STABILIZATION
}

\author{
DAVID EISENBUD AND BERND ULRICH
}

(Communicated by Harm Derksen)

\begin{abstract}
When $M$ is a finitely generated graded module over a standard graded algebra $S$ and $I$ is an ideal of $S$, it is known from work of Cutkosky, Herzog, Kodiyalam, Römer, Trung and Wang that the Castelnuovo-Mumford regularity of $I^{m} M$ has the form $d m+e$ when $m \gg 0$. We give an explicit bound on the $m$ for which this is true, under the hypotheses that $I$ is generated in a single degree and $M / I M$ has finite length, and we explore the phenomena that occur when these hypotheses are not satisfied. Finally, we prove a regularity bound for a reduced, equidimensional projective scheme of codimension 2 that is similar to the bound in the Eisenbud-Goto conjecture, under the additional hypotheses that the scheme lies on a quadric and has nice singularities.
\end{abstract}

\section{INTRODUCTION}

Let $S$ be a standard graded algebra over a field $k$, that is, an algebra generated by finitely many forms of degree one, let $M$ be a finitely generated graded $S$-module, and let $I$ be a homogeneous ideal not contained in the radical of ann $M$. If $H$ is an Artinian $S$-module, we set $\operatorname{reg} H=\max \left\{d \mid H_{d} \neq 0\right\}$ and we write $\operatorname{reg} M$ for the Castelnuovo-Mumford regularity

$$
\operatorname{reg} M=\operatorname{reg}_{S_{+}} M:=\max \left\{\operatorname{reg} H_{S_{+}}^{i}(M)+i\right\} .
$$

Combining results of Cutkosky-Herzog-Trung [C-H-T], Kodiyalam [Kod], Römer [ R and Trung-Wang [T-W], we have:

Theorem 0.1. There exist integers $m_{0}=m_{0}(I, M), d=d(I, M)$ and $e=e(I, M)$ such that for all $m \geq m_{0}$,

$$
\operatorname{reg} I^{m} M=d m+e .
$$

Furthermore, $d$ is the asymptotic generator degree of $I$ on $M$, i.e., the minimal number such that if $J \subset I$ is the ideal generated by the elements of $I$ of degree $\leq d$, then $I+$ ann $M$ is integral over $J+$ ann $M$.

This beautiful result begs for an answer to several questions: What is the significance of the number $e$ ? What is a reasonable bound $m_{0}$ ? What is the nature of the function $m \mapsto \operatorname{reg} I^{m} M$ for $m<m_{0} \ldots$ ? In general very little is known. But the result of the first section of this paper gives a value for $m_{0}$ in case

(*) $\quad I$ is generated in a single degree and $M / I M$ has finite length.

Received by the editors January 3, 2011

2010 Mathematics Subject Classification. Primary 13D02, 13C99, 13P20, 14 N05.

(C)2011 American Mathematical Society 
Here is a summary of our knowledge in this case. Under the hypothesis $(*)$ one has:

- The number $d$ in Theorem 0.1 is equal to the common degree of the generators of $I$.

- The differences $e_{m}:=\operatorname{reg} I^{m}-d m$ form a weakly decreasing sequence of nonnegative integers.

- The asymptotic value $e$ of the $e_{m}$ can be identified with the regularity of the restriction of the sheaf associated to $\mathrm{S}$ to the fibers of the morphism defined by $I$.

- If $S$ is a polynomial ring and $I$ is not a complete intersection, then the numbers $e_{m}$ are equal to the asymptotic value $e$ for all $m \geq m_{0}$, where $m_{0}$ is the $(0,1)$-regularity (defined below) of the Rees algebra $\mathcal{R}(I)$.

The first item in this list is immediate from Theorem 0.1 . The next two are proved in Eisenbud-Harris [E-H]. The last is the subject of the first section of this paper, where we also derive a sharper but more technical bound that is often optimal. We note that a different (somewhat larger) value for $m_{0}$ was proposed in CutkoskyHerzog-Trung $\mathrm{C}-\mathrm{H}-\mathrm{T}$, but the proof given was incomplete, as the authors of that paper have pointed out. Also, a bound similar to ours has been shown by Marc Chardin (private communication) with a spectral sequence proof.

In connection with the second item of the list, we observed in many cases that the sequence of first differences of the $e_{m}-e_{m+1}$ is also weakly decreasing. Is this always the case, under the assumption of $(*)$ ?

A key definition in this development is the $(0,1)$ (Castelnuovo-Mumford) regularity of the Rees module $\mathcal{R}(I . M)$. To define it, we recall that the Rees ring of $I$ is

$$
\mathcal{R}(I):=\bigoplus_{j \geq 0} I^{j} \cong \bigoplus_{j \geq 0} I^{j} t^{j}=S[I t] \subset S[t] .
$$

This ring is an epimorphic image of the polynomial $\operatorname{ring} T:=S\left[y_{1}, \ldots, y_{r}\right]$ via the map of $S$-algebras sending the $y_{i}$ to $t$ times the homogeneous minimal generators of $I$. In fact, this becomes a map of bigraded $k$-algebras if we set $\operatorname{deg} x_{i}=(1,0)$ and $\operatorname{deg} y_{i}=(0,1)$ (note that this is only possible because the generator degrees of $I$ are assumed to be equal). Next, if $M$ is a finitely generated graded $S$-module, we define

$$
\mathcal{R}(I, M):=S[I t] M \subset M \otimes_{S} S[t],
$$

which is a finitely generated bigraded module over $\mathcal{R}(I)$ and hence over $T$. Thus we consider a bigraded minimal free resolution

$$
\cdots \quad F_{1} \longrightarrow F_{0} \longrightarrow \mathcal{R}(I, M) \rightarrow 0
$$

of $\mathcal{R}(I, M)$ as a $T$-module, and we define the $(0,1)$-regularity $\operatorname{reg}_{\left(y_{1}, \ldots, y_{r}\right)} \mathcal{R}(I, M)$ to be the maximum integer $j$ such that $F_{i}$ has a free summand of the form $T(-a,-i-j)$ for some $i$ and $a$. As with the usual Castelnuovo-Mumford regularity, there is also a definition in terms of local cohomology, which we will use freely; see Römer $[$ ] for a detailed treatment.

In the second section of this paper, we turn to the question of what happens if we weaken the hypothesis $(*)$ to allow ideals that are not necessarily generated in a single degree. We found it surprisingly hard to give formulas for the numbers $e_{m}(I, M):=\operatorname{reg} I^{m} M-d(I, M) m$, even in very special cases; but we are able to provide such a formula when $M=S=k\left[x_{1}, \ldots, x_{n}\right]$ is a polynomial ring and 
$I=J+\left(x_{1}, \ldots, x_{n}\right)^{D}$ for some $D$, with $J$ an $\left(x_{1}, \ldots, x_{n}\right)$-primary ideal generated in a single degree, in terms of the numbers $e_{m}(J, M)$. In particular, we find that in this situation the numbers $e_{m}(I, M)-e_{m+1}(I, M)$ need not be weakly decreasing.

Section 3 of the paper uses some of the same ideas to prove a result close in spirit to the Eisenbud-Goto conjecture. Let $I \subset\left(x_{1}, \ldots, x_{n}\right)^{2}$ be a reduced, equidimensional homogeneous ideal in $S$, and suppose that $k$ is algebraically closed. The Eisenbud-Goto conjecture then asserts the following: if the projective variety $X$ associated to $I$ is connected in codimension 1 , then $\operatorname{reg} I \leq \operatorname{deg} X-\operatorname{codim} X+1$. This conjecture is wide open, even for smooth varieties $X$, when the dimension of $X$ is large.

In the conjecture the hypothesis "connected in codimension 1" is necessary, as an example of Giaimo (included in Section 3) shows; without the hypothesis, one must expect exponentially large regularity in general. But we are able to prove a bound that is only slightly weaker than that of the Eisenbud-Goto conjecture without any connectedness hypothesis, assuming instead that $X$ has codimension 2, lies on a quadric, and has only isolated "bad" singularities.

\section{1. m-PRIMARY IDEALS GENERATED IN ONE DEGREE}

In this section, $S$ denotes a standard graded algebra over a field $k$. We write $\mathfrak{m}$ for the homogeneous maximal ideal of $S$. Let $I \subset S$ be a homogeneous ideal generated in a single degree $d$.

We consider the Rees ring $\mathcal{R}(I)=S[I t]$ of $I$, a standard bigraded $k$-algebra as described above. Let $A$ be the ring

$$
A:=k\left[I_{d} t\right]=\bigoplus_{j} \mathcal{R}(I)_{(0, j)} \subset \mathcal{R}(I) .
$$

It is a bigraded subalgebra of $\mathcal{R}(I)$, generated in degree $(0,1)$, which is a direct summand as an $A$-module. We regard $A$ as a standard graded algebra, generated in degree 1 over $k$. We write $\mathfrak{n}$ for the homogeneous maximal ideal of $A$. Since $I$ is generated in one degree, $A$ is isomorphic to the special fiber ring $\mathcal{F}(I):=\mathcal{R}(I) \otimes_{S} k$.

For $M$ a finitely generated graded $S$-module we consider the Rees module $\mathcal{R}(I, M)=S[I t] M$, which is a finitely generated bigraded $\mathcal{R}(I)$-module. We define

$$
N_{i}(I, M):=k\left[I_{d} t\right] M_{i} \subset \mathcal{R}(I, M) .
$$

With the $(0,1)$-grading, the $A$-module $N_{i}(I, M)$ is generated in degree 0 and has degrees determined by the powers of $t$. As an $A$-module, $\mathcal{R}(I, M)$ is isomorphic to the direct sum of the $N_{i}(I, M)$. In particular,

$$
\operatorname{reg}_{\left(y_{1}, \ldots, y_{r}\right)} \mathcal{R}(I, M)
$$

the $(0,1)$-regularity of $\mathcal{R}(I, M)$, is the maximum of the regularities of the $N_{i}(I, M)$ (as $A$-modules). We shall see later how to restrict the range of $i$ required.

Theorem 1.1. Suppose that $I \subset S$ is an ideal generated by forms of a single degree $d$, and $M$ is a finitely generated graded $S$-module, generated in a single degree, such that $M / I M$ has finite length but $M$ does not. Let e be the number such that

$$
\operatorname{reg} I^{m} M=m d+e
$$

for $m \gg 0$. Let $N_{e}:=N_{e}(I, M)$. 
(1) The equality $\operatorname{reg} I^{m} M=m d+e$ holds if

$$
m \geq \max \left\{\operatorname{reg} H_{\mathfrak{n}}^{1}\left(N_{e}\right)+1, \frac{\operatorname{reg} M-e+1}{d}\right\} .
$$

(2) In case $\operatorname{reg} H_{\mathfrak{n}}^{1}\left(N_{e}\right) \geq(\operatorname{reg} M-e+1) / d$ and $m \geq 1$, the equality $\operatorname{reg} I^{m} M=$ $m d+e$ holds if and only if

$$
m \geq \operatorname{reg} H_{\mathfrak{n}}^{1}\left(N_{e}\right)+1 .
$$

Corollary 1.2. Let $I, S, M, d$, e be as in Theorem 1.1, and assume that $M$ is generated in degree 0 . The equality reg $I^{m} M=m d+e$ holds for all $m \geq$ $\max \left\{\operatorname{reg}_{\left(y_{1}, \ldots, y_{r}\right)} \mathcal{R}(I, M), \frac{\operatorname{reg} M+1}{d}\right\}$.

Proof of the Corollary. Since $N_{e}$ is an A-direct summand of $\mathcal{R}(I, M)$,

$$
\operatorname{reg} H_{\mathfrak{n}}^{1}\left(N_{e}\right)+1 \leq \operatorname{reg} N_{e} \leq \operatorname{reg}_{\left(y_{1}, \ldots, y_{r}\right)} \mathcal{R}(I, M) .
$$

Proof of the Theorem. After a shift of degree we may assume that $M$ is generated in degree 0. Consider first part (1), and assume that

$$
m \geq \max \left\{\operatorname{reg} H_{\mathfrak{n}}^{1}\left(N_{e}\right)+1, \frac{\operatorname{reg} M-e+1}{d}\right\} .
$$

By Eisenbud-Harris E-H], Proposition 1.1, $\left\{e_{n}\right\}$ is a nonincreasing sequence of nonnegative integers. Thus it suffices to show that $\operatorname{reg} I^{m} M \leq m d+e$. Our assumption on $m$ implies that reg $M \leq m d+e-1$. Because of the exact sequence

$$
0 \rightarrow I^{m} M \rightarrow M \rightarrow M / I^{m} M \rightarrow 0
$$

we only need to show that $\operatorname{reg} M / I^{m} M \leq m d+e-1$. Since $M / I^{m} M$ has finite length, this is equivalent to the statement that

$$
\left(I^{m} M\right)_{m d+e}=M_{m d+e} .
$$

The definition of $e$ implies, by the same argument, that this equality at least holds for sufficiently large $m$.

Let $N_{e}^{\prime}:=N_{e}\left(\mathfrak{m}^{d}, M\right)=\bigoplus_{j>0} M_{j d+e} t^{j}$, where the last equality holds because $M$ is generated in degree $0 \leq e$. Note that $N_{e}^{\prime}$ is naturally a graded $A$-module (with $j$-th graded piece $M_{j d+e} t^{j}$ ) and that $N_{e}$ is a submodule. Let

$$
E:=N_{e}^{\prime} / N_{e}=\frac{\bigoplus_{j \geq 0} M_{j d+e} t^{j}}{\bigoplus_{j \geq 0}\left(I^{j}\right)_{j d} M_{e} t^{j}} .
$$

By the preceding remark, the module $E$ has finite length.

We wish to show that $E_{m}=0$. Since $m \geq \operatorname{reg} H_{\mathfrak{n}}^{1}\left(N_{e}\right)+1$ we see from the exact sequence

$$
\cdots \rightarrow H_{\mathfrak{n}}^{0}\left(N_{e}^{\prime}\right) \rightarrow E \rightarrow H_{\mathfrak{n}}^{1}\left(N_{e}\right) \rightarrow H_{\mathfrak{n}}^{1}\left(N_{e}^{\prime}\right) \rightarrow \cdots
$$

that it suffices to prove $H_{\mathfrak{n}}^{0}\left(N_{e}^{\prime}\right)_{m}=0$.

We may identify the $A$-module $N_{e}^{\prime}$ with the $k\left[I_{d}\right]$-module $\bigoplus M_{d j+e}$, which is a $k\left[I_{d}\right]$-direct summand of $M$. Note that this identification sends the degree $j$ part of $N_{e}^{\prime}$ to the degree $d j+e$ part of $M$. Moreover, since $I_{d} S=I$ contains a power of $\mathfrak{m}$, the module $H_{\mathfrak{n}}^{0}\left(N_{e}^{\prime}\right)$ is a summand of $H_{\mathfrak{m}}^{0}(M)$ (with the same degree shift). On the other hand, $H_{\mathfrak{m}}^{0}(M)_{d j+e}=0$ when $d j+e \geq \operatorname{reg} M+1$. Thus $H_{\mathfrak{n}}^{0}\left(N_{e}^{\prime}\right)_{j}=0$ when $j \geq(\operatorname{reg} M-e+1) / d$, concluding the proof of part (1). 
We now consider part (2). Given part (1) and Eisenbud-Harris [E-H], Proposition 1.1, it suffices to show that if $m=\operatorname{reg} H_{\mathfrak{n}}^{1}\left(N_{e}\right)$, then $\operatorname{reg} I^{m} M \geq m d+e+1$. It follows from the hypothesis of part (2) that reg $M \leq m d+e-1$. Because of the exact sequence (1) we only need to show that $\operatorname{reg}\left(M / I^{m} M\right) \geq m d+e$. Let $N_{e}^{\prime}$ and $E$ be as in the proof of part (1). We want to show that $E_{m} \neq 0$.

Using the exact sequence (2) and the fact that $H_{\mathfrak{n}}^{1}\left(N_{e}\right)_{m} \neq 0$, we see that it suffices to show that $H_{\mathfrak{n}}^{1}\left(N_{e}^{\prime}\right)_{m}=0$. Since $N_{e}^{\prime}$ is a summand of $M$ (with a shift of degree) it suffices to show that $H_{\mathfrak{m}}^{1}(M)_{m d+e}=0$. This holds because, by hypothesis, $\operatorname{reg} M \leq m d+e-1$.

Conjecture. If $I, S, M$ are as in Theorem 1.1 and $M$ is generated in degree 0 , then the regularity of $N_{i}$ is nonincreasing from $i=0$. In particular, the $(0,1)$-regularity of $\mathcal{R}(I)$ is equal to the regularity of $k\left[I_{d}\right]$.

We can prove the conjecture in the case where $I$ is a power of the maximal ideal.

Proposition 1.3. Let $M$ be a finitely generated graded $S$-module, generated in degree 0 .

(1) If $i \geq 0$, then

$$
\operatorname{reg} N_{i}\left(\mathfrak{m}^{d}, M\right) \leq \max \left\{0, \frac{\operatorname{reg} M-i+(d-1) \operatorname{dim} M}{d}\right\}
$$

In particular reg $N_{i}\left(\mathfrak{m}^{d}, M\right)=0$ for $i \geq \operatorname{reg} M+(d-1)(\operatorname{dim} M-1)$.

(2) If $H_{\mathfrak{m}}^{0}(M)=0$, then the sequence of numbers $\left\{\operatorname{reg} N_{i}\left(\mathfrak{m}^{d}, M\right) \mid i \geq 0\right\}$ is weakly decreasing.

Proof. In the previous proof we have seen that there is a homogeneous isomorphism of $k\left[S_{d}\right]$-modules

$$
N_{i}:=N_{i}\left(\mathfrak{m}^{d}, M\right) \cong M_{i} K\left[S_{d}\right](i)=\bigoplus_{j \geq 0} M_{d j+i}=\left(M(i)_{\geq 0}\right)^{(d)},
$$

where we consider $N_{i}$ as a $k\left[S_{d}\right]$-module via the identification $k\left[S_{d} t\right] \cong k\left[S_{d}\right]$; here ${ }^{\left({ }^{(d)}\right.}$ denotes the Veronese functor.

The exact sequence

$$
0 \rightarrow M(i)_{\geq 0} \rightarrow M(i) \rightarrow M(i) / M(i)_{\geq 0} \rightarrow 0
$$

gives rise to an exact sequence

$$
\begin{gathered}
0 \rightarrow H_{\mathfrak{m}}^{0}\left(M(i)_{\geq 0}\right) \rightarrow H_{\mathfrak{m}}^{0}(M(i)) \rightarrow M(i) / M(i)_{\geq 0} \\
\quad \rightarrow H_{\mathfrak{m}}^{1}\left(M(i)_{\geq 0}\right) \rightarrow H_{\mathfrak{m}}^{1}(M(i)) \rightarrow 0
\end{gathered}
$$

and isomorphisms $H_{\mathfrak{m}}^{\ell}\left(M(i)_{\geq 0}\right) \cong H_{\mathfrak{m}}^{\ell}(M(i))$ for $2 \leq \ell$. 
Since the $d$-th Veronese functor commutes with taking local cohomology, it follows that

(3)

$$
\begin{aligned}
& \operatorname{reg}\left(M(i)_{\geq 0}\right)^{(d)} \\
& \leq \max \left\{1+\operatorname{reg}\left(M(i) / M(i)_{\geq 0}\right)^{(d)}, \max \left\{\operatorname{reg}\left(H_{\mathfrak{m}}^{\ell}(M(i))\right)^{(d)}+\ell \mid 0 \leq \ell \leq \operatorname{dim} M\right\}\right\} \\
& \leq \max \left\{0, \max \left\{\left\lfloor\frac{\operatorname{reg} H_{\mathfrak{m}}^{\ell}(M)-i}{d}\right\rfloor+\ell \mid 0 \leq \ell \leq \operatorname{dim} M\right\}\right\} \\
& \leq \max \left\{0, \max \left\{\left\lfloor\frac{\operatorname{reg} M-i-\ell}{d}\right\rfloor+\ell \mid 0 \leq \ell \leq \operatorname{dim} M\right\}\right\} \\
& \left.\leq \max \left\{0, \mid \frac{\operatorname{reg} M-i+(d-1) \operatorname{dim} M}{d}\right\rfloor\right\},
\end{aligned}
$$

which gives the desired formula. If $H_{\mathrm{m}}^{0}(M)=0$ and $M \neq 0$, then the first two inequalities are equalities, which implies part (2).

We can also prove the above conjecture for $i \geq e$, at least when $H_{\mathfrak{m}}^{0}(M)=0$.

Proposition 1.4. Suppose that $I \subset S$ is an ideal generated by forms of a single degree $d$, and $M \neq 0$ is a finitely generated graded $S$-module, generated in a single degree, such that $M / I M$ has finite length and $H_{\mathfrak{m}}^{0}(M)=0$. For each $m$, let $e_{m}$ be the number such that $\operatorname{reg} I^{m} M=m d+e_{m}$, and let $e:=e_{m}$ for $m \gg 0$. Let $N_{i}:=N_{i}(I, M)$ be the module defined above.

(1) $e_{m} \geq e_{m+1} \geq e_{m}-d$.

(2) If $i \geq e$, then $\operatorname{reg} N_{i+1} \leq \operatorname{reg} N_{i}$.

Proof. Again, after a shift of degree we may assume that $M$ is generated in degree 0 . The inequality $e_{m} \geq e_{m+1}$ of part (1) is proven in Eisenbud-Harris [E-H], Proposition 1.1.

For the second inequality it suffices to prove that $\operatorname{reg} I^{m} M \leq \operatorname{reg} I^{m+1} M$, for then $d m+e_{m} \leq d(m+1)+e_{m+1}$, that is, $e_{m} \leq d+e_{m+1}$.

Recall that $M / I^{m+1} M$ has finite length and $H_{\mathrm{m}}^{0}(M)=0$. The exact sequence

$$
0 \rightarrow I^{m+1} M \rightarrow M \rightarrow M / I^{m+1} M \rightarrow 0
$$

shows that $\operatorname{reg} H_{\mathfrak{m}}^{1}\left(I^{m+1} M\right)=\max \left\{\operatorname{reg} M / I^{m+1} M, \operatorname{reg} H_{\mathfrak{m}}^{1}(M)\right\}$ and moreover $H_{\mathfrak{m}}^{\ell}\left(I^{m+1} M\right)=\operatorname{reg} H_{\mathfrak{m}}^{\ell}(M)$ for $\ell \geq 2$. The same equalities hold for $I^{m} M$ in place of $I^{m+1} M$. The epimorphism of finite length modules $M / I^{m+1} M \rightarrow M / I^{m} M$ implies that reg $M / I^{m+1} M \geq \operatorname{reg} M / I^{m} M$, and the desired inequality follows.

For part (2), we note that for $i \geq e$ we can embed $N_{i}$ into $N_{i}^{\prime}:=N_{i}\left(\mathfrak{m}^{d}, M\right)$ with finite length cokernel and that $N_{i}$ is a submodule of $M$ (with a shift of degree); see the proof of Theorem 1.1(1). From $H_{\mathfrak{m}}^{0}(M)=0$ we deduce $H_{\mathfrak{m}}^{0}\left(N_{i}^{\prime}\right)=0$ and thus $H_{\mathfrak{m}}^{0}\left(N_{i}\right)=0$. Therefore $\operatorname{reg} N_{i}=\max \left\{\operatorname{reg} N_{i}^{\prime}, \operatorname{reg}\left(N_{i}^{\prime} / N_{i}\right)+1\right\}$.

Since $H_{\mathfrak{m}}^{0}(M)=0$, part (2) of Proposition 1.3 shows that the numbers reg $N_{i}^{\prime}$ are weakly decreasing. On the other hand, the generators of $\mathfrak{m}$ provide a homogeneous epimorphism $\bigoplus N_{i}^{\prime} \rightarrow N_{i+1}^{\prime}$ that induces an epimorphism $\bigoplus N_{i}^{\prime} / N_{i} \rightarrow N_{i+1}^{\prime} / N_{i+1}$. Thus the $(0,1)$-regularity of the finite length module $N_{i}^{\prime} / N_{i}$ is also weakly decreasing when $i \geq e$. 
Corollary 1.5. Let $S=k\left[x_{1}, \ldots, x_{n}\right]$ be a polynomial ring and let $I, d, e$ be as in Theorem 1.1. If $e=0$ and $m \geq \operatorname{reg} k\left[I_{d}\right]$, then $\operatorname{reg} I^{m}=m d+e$.

Proof. One uses Theorem 1.1(1).

Example 1.6. The regularity of $\mathcal{R}(I)$ is often much larger than the regularity of the module $N_{e}$. For the ideal $I=\left(x^{20}, x^{3} y^{17}, x^{12} y^{8}, y^{20}\right) \subset k[x, y]$ we have reg $I^{m} \geq 20 m+7$, with equality if and only if $m \geq 2$. Here the $(0,1)$-regularity of the Rees algebra, and also the regularity of $k\left[I_{d}\right]$, are equal to 7 . By Theorem [1.1. reg $H_{\mathfrak{n}}^{1}\left(N_{e}\right) \leq 1$ (and in fact equality holds). Now Proposition 1.3(1) shows that $\operatorname{reg} N_{e}=2$.

For the ideal $I=\left(x^{20}, x^{3} y^{17}, x^{15} y^{5}, y^{20}\right) \subset k[x, y]$ we have $\operatorname{reg} I^{m} \geq 20 m+$ 4 , with equality if and only if $m \geq 4$. Here again the $(0,1)$-regularity of the Rees algebra, and also the regularity of $k\left[I_{d}\right]$, are equal to 7 . By Theorem 1.1 . reg $H_{\mathfrak{n}}^{1}\left(N_{e}\right) \leq 3$ (and again, in fact, equality holds), and then reg $N_{e}=4$ according to Proposition 1.3(1).

\section{IDEALS WITH GENERATORS IN MORE THAN ONE DEGREE}

As a first example, we have:

Proposition 2.1. Let $I \subset S=k\left[x_{1}, \ldots, x_{n}\right]$ be a homogeneous ideal and let $M$ be a finitely generated graded $S$-module. If $I \subset S$ is generated by an $M$-regular sequence of degrees $d=d_{1} \geq \cdots \geq d_{t}$ and $m \geq 1$, then $\operatorname{reg} I^{m} M=d m+e$, where $e=\operatorname{reg} M+\sum_{i=2}^{t}\left(d_{i}-1\right)$.

Proof. Since $I$ is generated by a regular sequence on $M$, we may tensor $M$ with the Eagon-Northcott resolution of $I^{m}$ and get a resolution of $I^{m} \otimes M=I^{m} M$ by shifted copies of $M$. Analyzing the shifts, we see that $\operatorname{reg} I^{m} M=d m+e$.

Corollary 2.2. Let $I \subset S=k\left[x_{1}, \ldots, x_{n}\right]$ be a homogeneous ideal, and $M$ a finitely generated graded $S$-module. Let d be the asymptotic generator degree of $I$ on $M$, and write reg $I^{m} M=d m+e_{m}$. If I contains an $M$-regular sequence of degrees $d=d_{1} \geq \cdots \geq d_{t}$ with $t=\operatorname{dim} M$, then $e_{m} \leq \operatorname{reg} M+\sum_{i=2}^{t}\left(d_{i}-1\right)$ for every $m \geq 1$.

In general, we can analyze only special cases.

Theorem 2.3. Let $J \subset S=k\left[x_{1}, \ldots, x_{n}\right]$ be an $\mathfrak{m}$-primary ideal generated by forms of a single degree $d$. Write $I:=J+\mathfrak{m}^{d+k}$ for some $k \geq 0$. Let $f_{m}(p):=(d+k) m-k p$ and

$$
p_{m}:=\min \left\{p \geq 0 \mid \operatorname{reg} J^{p} \geq f_{m}(p)\right\} .
$$

For $m \geq 1$ we have

$$
\operatorname{reg} I^{m}=\min \left\{\operatorname{reg} J^{p_{m}}, f_{m}\left(p_{m}-1\right)\right\} .
$$

Proof. Define $e_{p}$ by the formula reg $J^{p}=d p+e_{p}$. Note that $p_{m}$ is finite, and in fact $p_{m} \leq m$ since reg $J^{m} \geq d m$.

We have

$$
I^{m}=\sum_{p=0}^{m} J^{p}\left(\mathfrak{m}^{d+k}\right)^{m-p} .
$$


Thus, $\operatorname{reg} I^{m} \leq \min \left\{\operatorname{reg} J^{p}\left(\mathfrak{m}^{d+k}\right)^{m-p} \mid 0 \leq p \leq m\right\}$. Moreover, $J^{p}\left(\mathfrak{m}^{d+k}\right)^{m-p}=$ $\left(J^{p}\right)_{\geq d p+(d+k)(m-p)}=\left(J^{p}\right)_{\geq f_{m}(p)}$, so

$$
\operatorname{reg} J^{p}\left(\mathfrak{m}^{d+k}\right)^{m-p}=\max \left\{\operatorname{reg} J^{p}, f_{m}(p)\right\} .
$$

We claim that the minimum value of $\operatorname{reg} J^{p}\left(\mathfrak{m}^{d+k}\right)^{m-p}$ is taken on either for $p=p_{m}$ or $p=p_{m}-1$, and that in either case it is

$$
\min _{0 \leq p \leq m}\left\{\operatorname{reg} J^{p}\left(\mathfrak{m}^{d+k}\right)^{m-p}\right\}=\min \left\{\operatorname{reg} J^{p_{m}}, f_{m}\left(p_{m}-1\right)\right\}
$$

This follows because, as $p$ increases, the function reg $J^{p}$ is weakly increasing (see the proof of Proposition 1.4 (1)) while $f_{m}(p)$ is decreasing, and for $p=m$ the first is at least as large as the second, and $p_{m} \geq 1$ (except when $I=S$ ); see Figure 1 . Note that the minimum value is the value claimed in the theorem for $\operatorname{reg} I^{m}$.

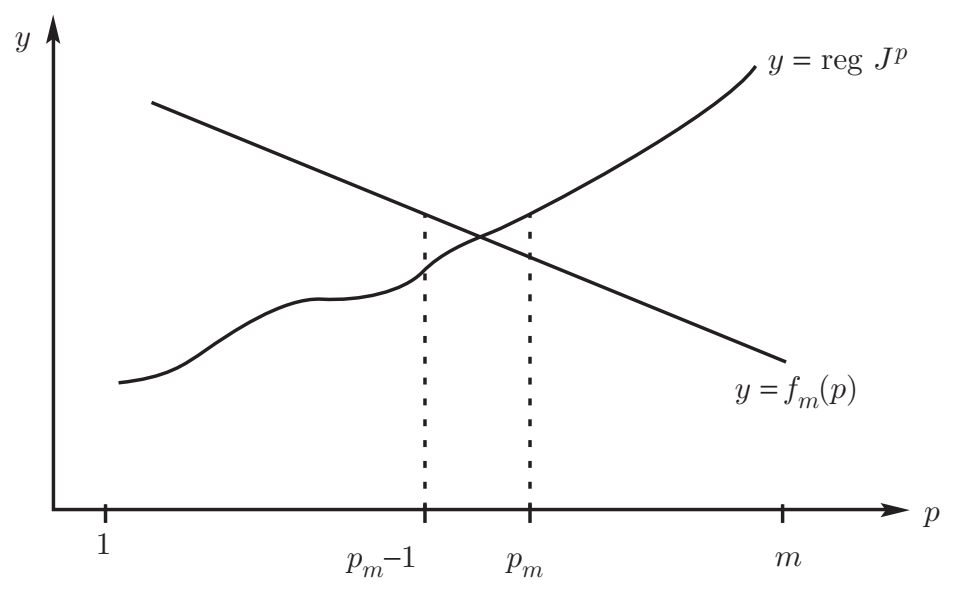

FiguRE 1. Where the graphs of $f_{m}(p)$ and reg $J^{p}$ cross

Thus it is enough to show that

$$
\operatorname{reg} I^{m} \geq \min \left\{\operatorname{reg} J^{p_{m}}, f_{m}\left(p_{m}-1\right)\right\} .
$$

Write $a:=\min \left\{\operatorname{reg} J^{p_{m}}, f_{m}\left(p_{m}-1\right)\right\}$. Note that $I^{m} \subset J^{p_{m}}+\mathfrak{m}^{f_{m}\left(p_{m}-1\right)}$. Thus it suffices to prove that

$$
\mathfrak{m}^{a-1} \not \subset J^{p_{m}}+\mathfrak{m}^{f_{m}\left(p_{m}-1\right)} .
$$

Since $a-1<f_{m}\left(p_{m}-1\right)$, this is equivalent to $\mathfrak{m}^{a-1} \not \subset J^{p_{m}}$. But the latter holds because $a-1<\operatorname{reg} J^{p_{m}}$.

Example 2.4. If $I$ is not generated in a single degree, then in the formula reg $I^{m}=$ $m d+e_{m}$ the $e_{m}$ may not be weakly decreasing. They can even go up and then down. For example, using Theorem 2.3 one can easily compute that if

$$
I=\left(x_{1}^{4}, \ldots, x_{4}^{4}\right)\left(x_{1}, \ldots, x_{4}\right)+\left(x_{1}, \ldots, x_{4}\right)^{6} \subset S=k\left[x_{1}, \ldots, x_{4}\right],
$$

then $\operatorname{reg} I^{m}=5 m+e_{m}$, where the successive values of $e_{m}$ for $m=1,2, \ldots$ are $1,2,2,1,1,1,1,1,0,0,0, \ldots$ 
Proposition 2.5. Let $I \subset S=k\left[x_{1}, \ldots, x_{n}\right]$ be a homogeneous ideal and let $M$ be a finitely generated graded $S$-module, concentrated in nonnegative degrees, such that $M / I M$ has finite length but $M$ does not. Let $d$ be the asymptotic generator degree of $I$ on $M$, and write $\operatorname{reg} I^{m} M=d m+e_{m}$.

(1) If I is generated in degrees $\leq d$, then the sequence of integers $\left\{e_{m} \mid m \geq\right.$ $(\operatorname{reg} M+1) / d\}$ is weakly decreasing.

(2) If the associated graded module $\mathfrak{g r}_{I}(M)$ has positive depth, then the sequence $\left\{e_{m} \mid m \geq(\operatorname{reg} M+1) / d\right\}$ is weakly increasing.

Proof. We first prove part (1). If $I$ is generated by homogeneous elements of degrees $d_{i}$, then multiplication by these elements gives a homogeneous surjection

$$
\bigoplus_{i}\left(\frac{I^{m-1} M}{I^{m} M}\left(-d_{i}\right)\right) \rightarrow \frac{I^{m} M}{I^{m+1} M}
$$

of modules of finite length. Thus

$$
\operatorname{reg} I^{m} M / I^{m+1} M \leq \operatorname{reg} I^{m-1} M / I^{m} M+d \leq \operatorname{reg} M / I^{m} M+d .
$$

Now the exact sequence

$$
0 \rightarrow I^{m} M / I^{m+1} M \rightarrow M / I^{m+1} M \rightarrow M / I^{m} M \rightarrow 0
$$

shows that $\operatorname{reg} M / I^{m+1} M \leq \operatorname{reg} M / I^{m} M+d$.

Since $\operatorname{reg}\left(I^{m}\right)^{p} M=(d m) p+e_{m p}$ for $p \gg 0$, we conclude that the asymptotic generator degree of $I^{m}$ on $M$ is $d m$. Thus the highest generator degree of $I^{m} M$ is at least $d m$ because $M$ is concentrated in nonnegative degrees. It follows that $\operatorname{reg} I^{m} M \geq d m$. Thus, if $m \geq(\operatorname{reg} M+1) / d$, then $\operatorname{reg} M \leq d m-1 \leq \operatorname{reg} I^{m} M-1$. Now the inequality $\operatorname{reg} M / I^{m+1} M \leq \operatorname{reg} M / I^{m} M+d$ implies that $\operatorname{reg} I^{m+1} M \leq$ $\operatorname{reg} I^{m} M+d$.

For part (2) we may assume that $k$ is infinite. The definition of $d$ shows that for some integer $p$ we have

$$
\left(I / I^{2}\right)^{p} \mathfrak{g r}_{I}(M) \subset\left(\left(I_{\leq d}+I^{2}\right) / I^{2}\right) \mathfrak{g r}_{I}(M) .
$$

It follows that there exists an element $a \in I_{d}$ whose leading form $a+I^{2} \in \mathfrak{g r}_{I}(S)$ is not a zero-divisor on $\mathfrak{g r}_{I}(M)$. Hence $I^{m+1} M:_{M} a=I^{m} M$. Thus multiplication by $a$ induces an embedding

$$
\frac{M}{I^{m} M}(-d) \hookrightarrow \frac{M}{I^{m+1} M} .
$$

This implies that $\operatorname{reg} M / I^{m+1} M \geq \operatorname{reg} M / I^{m} M+d$, and hence $\operatorname{reg} I^{m+1} M \geq$ $\operatorname{reg} I^{m} M+d$ whenever $m \geq \operatorname{reg} M / d$.

Corollary 2.6. Let $I \subset S=k\left[x_{1}, \ldots, x_{n}\right]$ be a homogeneous $\mathfrak{m}$-primary ideal with asymptotic generator degree $d$. If $I$ is generated in degrees $\leq d$ and $\mathfrak{g r}_{I}(S)$ has positive depth, then $\operatorname{reg} I^{m}=d m+e$ for some e and every $m \geq 1$.

Example 2.7. One cannot drop the assumption of generation in degree $\leq d$ from Corollary [2.6. If

$$
I=\left(x^{4}, y^{4}, z^{4}\right)+(x, y, z)^{5} \subset S=k[x, y, z],
$$

then $\operatorname{reg} I^{m}=4 m+e_{m}$, where the successive values of $e_{m}$ for $m=1,2, \ldots$ are $1,2,2,2,2, \ldots$ Computation with Macaulay2 shows that the depth of the associated graded ring of $I$ is positive. 


\section{A case of the (Almost) Eisenbud-Goto conjecture}

Eisenbud and Goto [E-G] conjecture that the regularity of a nondegenerate, geometrically reduced irreducible subscheme $X \subset \mathbb{P}^{n}$ has regularity at most $\operatorname{deg} X-$ $\operatorname{codim} X+1$. They further conjecture that the hypothesis can be weakened to say that the nondegenerate scheme is geometrically reduced and connected in codimension 1, and this has been proved by Giaimo G for curves. The bound can fail for disconnected schemes. For example, if $X$ is the union of two skew lines in $\mathbb{P}^{3}$, then the degree of $X$ is 2 but the regularity (that is, the regularity of the ideal of $X$ ) is 2 rather than 1. Derksen and Sidman [D-S have shown that in general a union of linear subspaces of projective space has regularity at most the number of subspaces.

One might guess from this that the regularity of a reduced equidimensional scheme would be bounded by the degree of the scheme, but this is not the case.

Example 3.1 (Giaimo, unpublished). Here is a reduced equidimensional union of two irreducible complete intersections whose regularity is much larger than its degree:

By Mayr-Meyer [M-M] there is a homogeneous ideal $I \subset S=\mathbb{C}\left[x_{1}, \ldots, x_{n}\right]$ generated by $10 n$ forms of degrees two and three, having regularity of the order of $2^{2^{n}}$. In the $\operatorname{ring} R=S\left[z_{1}, \ldots\right]$ we build an ideal $I^{\prime}$ whose generators correspond to those of $I$ by replacing the monomials in the generators of $I$ with products of new variables $z_{j}$ in such a way that each $z_{j}$ occurs only linearly, and no $z_{j}$ occurs twice. Clearly the generators of this new ideal are a regular sequence. If any of the generators are monomials, we add further new variables $w_{j}$ and make each a binomial that will be a prime. Since the variables are all distinct, the resulting complete intersection will also be prime, and modulo an ideal of the form $L=$ $\left(\left\{z_{j}-x_{p(j)}\right\}\right)+\left(\left\{w_{j}\right\}\right)$ the ideal $I^{\prime}$ becomes equal to the ideal $I$. The codimension of $L$ is clearly at least as big as the codimension of the complete intersection. We add further variables to the ambient ring and to the complete intersection $I^{\prime}$ to make the codimensions the same.

The ideal $I^{\prime} \cap L$ now defines the union of two reduced, irreducible complete intersections, while the ideal $I^{\prime}+L$ defines the same factor ring as the original Mayr-Meyer example. From the short exact sequence

$$
0 \rightarrow I^{\prime} \cap L \rightarrow I^{\prime} \oplus L \rightarrow I^{\prime}+L \rightarrow 0,
$$

we see that the regularity of $I^{\prime} \cap L$ is of the order of $2^{2^{n}}$. On the other hand, the degree of the subscheme defined by $I^{\prime} \cap L$ is at most of the order of $3^{10 n}$.

We state our result in terms of the regularity of the homogeneous coordinate ring $S_{X}$ of $X$, which is one less than $\operatorname{reg} X$, to emphasize the parallel between the two parts of the theorem. The first part of the theorem deals with the Eisenbud-Goto conjecture, whereas the second part is motivated by the estimate of Corollary 1.2 . Recall that a local algebra essentially of finite type over a field of characteristic zero is said to have a rational singularity if it is normal and Cohen-Macaulay and, if $\pi: \tilde{X} \rightarrow \operatorname{Spec} R$ is a resolution of singularities, then $\pi_{*}\left(\omega_{\tilde{X}}\right)=\omega_{\text {Spec } R}$.

Theorem 3.2. Let $X$ be a reduced equidimensional subscheme of codimension 2 in $\mathbb{P}_{k}^{n}$, where $k$ is a field of characteristic zero. Assume that $X$ lies on a quadric hypersurface and that the locus of nonrational singularities of $X$ has dimension at most zero. Let $S_{X}$ be the homogeneous coordinate ring of $X$. 
(1) $\operatorname{reg} S_{X} \leq \operatorname{deg} X$.

(2) If $x_{1}, \ldots, x_{n}$ are general linear forms in $S_{X}$, and $I$ is the ideal they generate, then $\operatorname{reg}_{\left(y_{1}, \ldots, y_{r}\right)} \mathcal{R}\left(I, S_{X}\right) \leq \operatorname{deg} X-\operatorname{codim} X+1$.

Note that the Eisenbud-Goto conjecture would say, under the additional hypothesis that $X$ is nondegenerate and connected in codimension 1, that reg $S_{X} \leq$ $\operatorname{deg} X-\operatorname{codim} X=\operatorname{deg} X-2$.

Proof. We make use of the notation introduced in part (2) of the theorem, and we write $\mathfrak{m}$ for the homogeneous maximal ideal of $S_{X}$. Let $\mathcal{F}:=k\left[I_{1}\right] \subset S_{X}$ and note that $\mathcal{F}$ is isomorphic to the special fiber ring $\mathcal{F} \cong \mathcal{R}\left(I, S_{X}\right) / \mathfrak{m} \mathcal{R}\left(I, S_{X}\right)$. Let $x$ be a linear form such that $\mathfrak{m}=(I, x)$. Because the $x_{1}, \ldots, x_{n}$ are general and the ideal defining $X$ contains a quadric, $S_{X}=\mathcal{F}+\mathcal{F} x$. Thus $S_{X} / \mathcal{F} \cong\left(\mathcal{F} /\left(\mathcal{F}: \mathcal{F} S_{X}\right)\right)(-1)$. The extension $\mathcal{F} \subset S_{X}$ is finite and birational. Hence $\mathcal{F}$ is the ring of a hypersurface whose degree is $\operatorname{deg} S_{X}$ in $\mathbb{P}^{n-1}$. It follows that $\operatorname{reg} \mathcal{F}=\operatorname{deg} S_{X}-1$.

As $\omega_{\mathcal{F}}=\mathcal{F}\left(-n+\operatorname{deg} S_{X}\right)$ we have $\mathcal{F}:_{\mathcal{F}} S_{X}=\operatorname{Hom}_{\mathcal{F}}\left(S_{X}, \mathcal{F}\right)=\omega_{S_{X}}(n-$ $\operatorname{deg} S_{X}$ ). The hypothesis that the characteristic is zero and that the equidimensional scheme $X$ has at most isolated nonrational singularities implies that the regularity of $\omega_{S_{X}}$ is at most $\operatorname{dim} S_{X}=n-1$ (see Chardin-Ulrich [C-U], Theorem 1.3, which is based on results of Ohsawa $\mathrm{O}$ and Kollár [Kol, Theorem 2.1(iii)). It follows that $\operatorname{reg}\left(\mathcal{F}:_{\mathcal{F}} S_{X}\right) \leq n-1-\left(n-\operatorname{deg} S_{X}\right)=\operatorname{deg} S_{X}-1$. Thus reg $S_{X} / \mathcal{F} \leq \operatorname{deg} S_{X}$, and therefore reg $S_{X} \leq \operatorname{deg} S_{X}$, proving the first statement.

For the second statement, let $G:=\mathfrak{g r}_{I}\left(S_{X}\right)$ be the associated graded ring of $S_{X}$ with respect to $I$, which is an $S_{X}$-module via the map $S_{X} \rightarrow S_{X} / I=G_{0}$. By Johnson and Ulrich [J-U], Proposition 4.1, one has $\operatorname{reg}_{\left(y_{1}, \ldots, y_{r}\right)} \mathcal{R}\left(I, S_{X}\right)=\operatorname{reg}_{\left(y_{1}, \ldots, y_{r}\right)} G$, so it suffices to bound the latter.

Note that $\mathcal{F}=G / \mathfrak{m} G=G / x G$. Because the ideal defining $X$ contains a quadric we have $x^{2} \in I$. It follows that $x^{2} G=0$. Of course $x G \cong G /\left(0:_{G} x\right)$. We will show that $G /\left(0:_{G} x\right) \cong \mathcal{F} /\left(\mathcal{F}:_{\mathcal{F}} S_{X}\right)$. Indeed, the embedding $\mathcal{F} \cong k\left[I_{1} t\right] \subset \mathcal{R}\left(I, S_{X}\right)$ induces a map $\mathcal{F} \rightarrow G /\left(0::_{G} x\right)$, which is surjective because $x G \subset 0:_{G} x$. To compute the kernel, let $f \in \mathcal{F}$ be a form of degree $i$. The image of $f x$ in $G$ is 0 if and only if, as elements of $S_{X}$, we have $f x \in I^{i+1}$. But the degree (in $S_{X}$ ) of $f x$ is $i+1$, so this happens if and only if $f x \in \mathcal{F}_{i+1}$. This in turn means that $f \in \mathcal{F}:_{\mathcal{F}} x=\mathcal{F}:_{\mathcal{F}} S_{X}$.

From the computation of the regularity of $\mathcal{F}:_{\mathcal{F}} S_{X}$ above, we get $\operatorname{reg} G \leq$ $\max \left\{\operatorname{reg} \mathcal{F} /\left(\mathcal{F}: \mathcal{F} S_{X}\right), \operatorname{reg} \mathcal{F}\right\}=\operatorname{deg} S_{X}-1$.

\section{REFERENCES}

[C-U] M. Chardin and B. Ulrich, Liaison and Castelnuovo-Mumford regularity, Amer. J. Math. 124 (2002), 1103-1124. MR1939782 (2004c:14095)

[C-H-T] S. Cutkosky, J. Herzog, and N.V. Trung, Asymptotic behavior of the CastelnuovoMumford regularity, Compositio Math. 118 (1999), 243-261. MR.1711319 (2000f:13037)

[D-S] H. Derksen and J. Sidman, A sharp bound for the Castelnuovo-Mumford regularity of subspace arrangements, Adv. Math. 172 (2002), 151-157. MR.1942401 (2003m:13013)

[E-G] D. Eisenbud and S. Goto, Linear free resolutions and minimal multiplicity, J. Algebra 88 (1984), 89-133. MR741934 (85f:13023)

[E-H] D. Eisenbud and J. Harris, Powers of ideals and fibers of morphisms, Math. Res. Lett. 17 (2010), 267-273. MR2644374 (2011e:14003)

[G] D. Giaimo, On the Castelnuovo-Mumford regularity of connected curves, Trans. Amer. Math. Soc. 358 (2006), 267-284. MR2171233(2006f:13013)

[Kod] V. Kodiyalam, Asymptotic behavior of Castelnuovo-Mumford regularity, Proc. Amer. Math. Soc. 128 (2000), 407-411. MR1621961 (2000c:13027) 
[J-U] M. Johnson and B. Ulrich, Artin-Nagata properties and Cohen-Macaulay associated graded rings, Compositio Math 103 (1996), 7-29. MR.1404996 (97f:13006)

[Kol] J. Kollár, Higher direct images of dualizing sheaves I, Ann. of Math. (2) 123 (1986), 11-42. MR825838 (87c:14038)

[M-M] E. Mayr and A. Meyer, The complexity of the word problems for commutative semigroups and polynomial ideals, Adv. Math. 46 (1982), 305-329. MR683204 (84g:20099)

[O] T. Ohsawa, Vanishing theorems on complete Kähler manifolds, Publ. Res. Inst. Math. Sci. 20 (1984), 21-38. MR736089 (85g:32046)

[R] T. Römer, Homological properties of bigraded algebras, Illinois J. Math. 45 (2001), 1361-1376. MR.1895463 (2003d:13015)

[T-W] N.V. Trung and H.-J. Wang, On the asymptotic linearity of Castelnuovo-Mumford regularity, J. Pure and Appl. Alg. 201 (2005), 42-48. MR2158746 (2006k:13039)

Department of Mathematics, University of California, Berkeley, Berkeley, CaliFORNIA 94720

E-mail address: eisenbud@math.berkeley.edu

Department of Mathematics, Purdue University, West Lafayette, Indiana 47907

E-mail address: ulrich@math.purdue.edu 